\title{
Beyond Viral Suppression: Quality of Life among stable ART Clients in a Differentiated Service Delivery Intervention in Tanzania
}

\section{Nwanneka Ebelechukwu Okere ( $\nabla$ nnekaokere@gmail.com )}

AIGHD: Amsterdam Institute for Global Health and Development https://orcid.org/0000-0001-9182-6518

\section{Veronica Censi}

Doctors With Africa (CUAMM)

Clementina Machibya

Ngokolo Health Centre Shinyanga

\section{Kathleen Costigan}

Bugisi Health Centre

\section{Patrobas Katambi}

Ngokolo Health Centre Shinyanga

Guilia Martelli

Doctors With Africa (CUAMM)

Josien de Klerk

Amsterdam Institute for Global Health and Development

\section{Sabine Hermans}

Amsterdam Institute for Global Health and Development

\section{Gabriela Gomez}

London School of Hygiene \& Tropical Medicine

\section{Anton Pozniak}

Chelsea and Westminster Hospital NHS Foundation Trust

\section{Tobias Rinke de Wit}

Amsterdam Institute for Global Health and Development

\section{Denise Naniche}

ISGLOBAL: Instituto de Salud Global de Barcelona

\section{Research}

Keywords: HIV (PLHIV), differentiated service delivery (DSD)

Posted Date: October 15th, 2020

DOI: https://doi.org/10.21203/rs.3.rs-91027/v1

License: (c) (1) This work is licensed under a Creative Commons Attribution 4.0 International License. Read Full License 
Version of Record: A version of this preprint was published at Quality of Life Research on May 30th, 2021. See the published version at https://doi.org/10.1007/s11136-021-02889-z. 


\section{Abstract}

Background: More people living with HIV (PLHIV) in resource-limited settings are virally suppressed and living longer, mainly due to an increased access to effective antiretroviral therapy (ART). With the expansion of ART programmes, the World Health Organisation recommended differentiated service delivery (DSD) as an alternative less resourcedemanding way of accessing HIV services. While maintaining quality of care and continued adherence among patients are health system's priorities, monitoring patient's quality of life is key to ensure sustainability and uptake of services. However, health-related quality of life (HRQoL) is understudied in sub-Saharan Africa (SSA). We aimed to assess HRQoL among stable ART clients accessing ART care in adherence clubs implemented in Tanzania.

Methods: We conducted a cross-sectional survey from May to August 2019 among stable ART clients randomly sampled among those accessing clinics and clubs daily in two sites, a rural and peri-urban setting in the Shinyanga region. HRQoL data was collected, after obtaining informed consent, using the interviewer-administered Functional Assessment of HIV infection (FAHI), a validated HIV-specific HRQoL instrument. We also collected on sociodemographic, HIV care and service accessibility factors. Descriptive analysis, modified Poisson regression with robust variance and a stepwise multiple linear regression were performed to analyse HRQoL and its determinants.

Results: A total of 629 participants were enrolled, of which $40 \%$ were DSD patients. DSD and clinic participants showed similar HRQoL scores [mean (SD), p value]; FAHI total [152.2 (22.2) vs 153.8 (20.6), p 0.687]. Accessibility factors, e.g. less time spent during and less frequent DSD meetings, contributed to emotional wellbeing among DSD participants compared to those in clinic $(53.4 \%$ vs $18.5 \%, p=<0.001)$. Satisfactory $(>80 \%$ of achievable) HRQoL scoring was independently associated with (relative risk [95\% confidence interval], p value) being male (1.18[1.06-1.31], p 0.002); being married (1.22 [1.01-1.45, p 0.043); and living in an urban setting (1.28 [1.09-1.49], p 0.001).

Conclusions: DSD does not appear to compromise HRQoL and, encouragingly, it could contribute to an improvement in emotional wellbeing among patients. While DSD shows promise in improving acceptability among patients and, therefore, sustainability of such services, our research highlights future research avenues to identify supporting interventions to improve other HRQoL domains among PLHIV.

\section{Background}

Access to effective antiretroviral therapy (ART) has contributed largely to an increased number of people living with HIV (PLHIV) being virally suppressed and living longer (1-4).However, the physical consequences of long-term exposure to ARVs has yet to be fully elucidated and evidence associates PLHIV on ART with an increased risk of cardiovascular disease, liver disease and various malignancies(5-8). While PLHIV report significantly lower health related quality of life (HRQoL) when compared to the general population in high income countries (HIC) (9), HRQoL among PLHIV is understudied in low and middle income countries (LMIC), especially in sub-Saharan Africa (SSA). With increasing number of PLHIV on ART who are aging in SSA, including a fourth 90 in the UNAIDS global HIV targets will ensure the much-needed monitoring of HRQoL $(10,11)$.

HRQoL is a multidimensional concept depicting an individual's subjective perception of current health status and outlook of the future (12,13). HRQoL studies assess individuals' perception of their health and how it affects or is affected by other aspects of life(13). Among PLHIV, ART impacted HRQoL positively, especially in LMIC when ART start was guided by CD4 thresholds $(1,14,15)$. Subsequent studies predicted factors associated with good HRQoL among PLHIV e.g. being married, absence of co-morbidities, higher education, living in an urban setting, status disclosure, being on ART longer, being employed, fewer pills and good adherence (16-21). Conversely, factors found to 
be associated with lower HRQoL included stigma, same-sex relationships, being symptomatic, illiteracy and not being sexually active $(18,19,22-24)$. HRQoL studies among virally suppressed PLHIV are limited in LMIC $(9,23,25)$.

In SSA, HRQoL studies have mostly been conducted among clients who access ART in clinical settings $(24,26-28)$. Differentiated service delivery (DSD) offer virally-suppressed PLHIV alternative models of HIV care both within or and outside of the clinic (29). Out-of-clinic models limit contact with the formal health system and rely upon community health workers (CHW) who are mostly trained volunteers for service delivery. Most evaluations of such models focused on adherence and quality of care yet changes in delivery models of care may also affect HRQoL.

With 1.6 million PLHIV and a prevalence of $4.6 \%$ among adults in 2018 , it is estimated that only $62 \%$ of PLHIV on ART in Tanzania are virally suppressed (30). Though studies show favorable patient-related outcomes with DSD interventions elsewhere, there is dearth of evidence within the Tanzanian context (31-33). Our study aimed to assess HRQoL among stable ART clients accessing ART care in a flagship Test and Treat (T\&T) project in north-western Tanzania. We compared HRQoL scores and determinants of HRQoL between stable ART clients receiving either standard clinic-based care or ART clubs DSD care.

\section{Methods}

\section{Study setting and population}

The T\&T project is hosted by the Catholic Diocese of Shinyanga which cover both Shinyanga and Simiyu regions in north-western Tanzania. Besides Shinyanga urban, Kahama urban and Bariadi districts, the regions are largely rural. Project sites are four HIV care and treatment centres (CTC) referred to as hubs, two hubs each in Shinyanga region (i.e. Ngokolo and Bugisi) and in Simiyu region (i.e. Songambele and Mwamapala). Data were collected from May to August 2019. Participants were recruited at the two hubs in Shinyanga region and their related ART clubs. Eligibility criteria included being adult $\geq 18$ years and stable on ART according to the Tanzanian guidelines: on ART 1st line regimen $\geq 6$-months, viral load $<50$ copies/ml and no current chronic illness (34). At the hubs, participants were randomly sampled from eligible clients expected for daily clinic appointment. Eligible participants were approached as they attended clinic appointments or club meetings. At the clubs, all members were approached as stability was an eligibility criterion for DSD participation. Those patients consenting completed the interviewer-administered questionnaire.

\section{Data Collection}

We used a HIV specific HRQoL tool which has been validated for low literacy Swahili population, the Kiswahili translation of the Functional Assessment of HIV Infection (FAHI) (35). Outcomes of interest were the total and domain specific FAHI scores. In the present study, Cronbach alpha was $0.68,0.73,0.67,0.71$ and 0.81 for the PWB, EWB, FGWB, SWB and CF domains respectively indicating acceptable internal consistency. The FAHI is a 47-item tool with five domains namely physical wellbeing (PWB) - 10 items, emotional wellbeing (EWB) - 10 items, functional \& global wellbeing (FGWB) - 13 items, social wellbeing (SWB)-8 items and cognitive functioning (CF) - 3 items (36). Scores ranged for each item between 1 and 4 . We derived (a) domain scores by summing respective item scores - ranges for PWB and EWB were 10 to 40, FGWB 13 to 52, SWB 8 to 32 and CF 3 to12; (b) total FAHI scores by summing all five domain scores - ranging between 44 and 176; (c) FAHI proportional score by calculating each individual score as a proportion of the maximum possible total or domain scores; and (d) a dichotomous (satisfactory/less than satisfactory FAHI HRQOL) variable for total and domains. We considered a score in the highest quintile i.e. $\geq 80 \%$ of FAHI total or domain scores as satisfactory. 
Secondary outcomes were factors associated with satisfactory HRQoL and domain scores. Three categories of additional data were collected to assess these factors: socio-demographic (location, sex, age, educational level, marital status, employment status and income level), HIV care (duration on ART, CD4 count at ART start and recency of viral load result) and service access (location, time spent during clinic visit/club meeting[wait time], time spent travelling to clinic/club[travel time] and frequency of service-delivery). Data entry, collation and cleaning was done using EpiData (37).

\section{Sample Size And Statistical Analysis}

Our sample size calculation was based on EQ-index scores and extrapolated to proportional FAHI scores. We assumed a difference in proportional scores of 0.10 (0.80 to 0.90) between clinic and DSD participants, a standard deviation of 0.40 as determined by Louwagie et al in South Africa and 10\% refusal rate requiring thus a minimum of 542 participants overall with 271 participants per service delivery group to have $80 \%$ power to reject the null hypothesis of no difference(1).

Categorical variables were presented as percentages and continuous variables as means ( \pm standard deviation) or medians ( \pm interquartile range) as appropriate. Comparisons between clinic and DSD participants were done using Mann Whitney or Kruskal Wallis tests. Association between socio-demographic, HIV care and service access factors and satisfactory FAHI HRQoL were examined using modified Poisson regression with robust variance which has been shown to give more accurate estimates than logistics regression(38). Sex, age, marital status, and variables showing significant bivariate association at $\mathrm{p}$-value of $<0.1$ were included in the multivariable model. A 3-step hierarchical multiple linear regression was used to quantify the contribution of the three factor categories to the variance of FAHI scores observed. Socio-demographic variables were entered the model in the first step, followed by HIV care variables and lastly, service access variables.

We examined variables for multicollinearity using tolerance values and variance inflation factor (VIF) statistics. We generated a Receiver Operative Characteristic (ROC) i.e. area under curve (AUC) to test the predictive ability of the model. We assessed the 33 and 28 missing observations dropped from the clinic and DSD in the step 3 hierarchical linear models respectively to observe any significant differences in mean FAHItotal. All analyses were performed using STATA software version 16.0.

Ethical approval for the study was obtained from the National Institute for Medical research (NIMR; approval number NIMR/HQ/R.8c/Vol. I/674).

\section{Results}

\section{Characteristics of study population}

Of 667 PLHIV approached to participate, 641 consented to participate (response rate of 96.1\%), and 629 were included in the final analysis (12 excluded due to non-eligibility). While overall majority of participants were female (63\%), there were significantly more men in clinic-based care compared to DSD, and clinic-based participants were also significantly older ( see Table 1). The mean numbers of years-on-ART and mean CD 4 count at ART-start were significantly longer (4.9 vs 4.1 years, $p<0.001$ ) and higher ( 398.1 vs 341.4 cells $\left./ \mathrm{mm}^{3}, p<0.001\right)$ for DSD participants. DSD participants reported shorter time spent on travel (84.7 vs $34.3 \mathrm{~min}, \mathrm{p}<0.001)$ and during club meetings (140.3 vs $83.8 \mathrm{~min}, \mathrm{p}<0.001)$. There were more DSD participants in the urban area $(34.1 \%$ vs $60.6 \%)$. Table 1 provides details on the characteristics of study participants according to service delivery mode. 
Table 1

Socio-demographic, HIV-related, and delivery mode-related characteristics according to service delivery mode.

\begin{tabular}{|c|c|c|c|}
\hline & $\begin{array}{l}\text { Clinic-based }(\mathrm{N}= \\
378)\end{array}$ & DSD $(N=251)$ & $\begin{array}{l}\text { p- } \\
\text { value* }\end{array}$ \\
\hline \multicolumn{4}{|c|}{ Sociodemographic information } \\
\hline Location, n (\%) & 324 (65.8) & 168 (34.1) & $<0.001$ \\
\hline - Bugisi (Rural) & $54(39.4)$ & $83(60.6)$ & \\
\hline \multicolumn{4}{|l|}{ - Ngokolo (Urban) } \\
\hline $\operatorname{Sex}(n, \%)$ & 224 (59.3) & $172(68.5)$ & 0.018 \\
\hline - Female & $154(40,7)$ & 79 (31.5) & \\
\hline \multicolumn{4}{|l|}{ - Male } \\
\hline Age in years, median (IQR) & $39.3(33.3-48.1)$ & $44.7(37.6-54.0)$ & $<0.001$ \\
\hline Age-groups, n, (\%) & $25(6.6)$ & $6(2.4)$ & $<0.001$ \\
\hline$-<25$ & $96(25.1)$ & $35(13.9)$ & \\
\hline$-25-34$ & $137(36.2)$ & $91(36.3)$ & \\
\hline$-35-44$ & $75(19.8)$ & $62(24.7)$ & \\
\hline$-45-54$ & $33(8.7)$ & $40(15.9)$ & \\
\hline$-55-64$ & $13(3.4)$ & $17(6.8)$ & \\
\hline \multicolumn{4}{|l|}{$-\geq 65$} \\
\hline Educational level, n (\%) & $97(25.7)$ & $60(23.9)$ & 0.744 \\
\hline - No education & $261(69.1)$ & $180(71.7)$ & \\
\hline - Primary & $20(5.3)$ & $11(4.4)$ & \\
\hline \multicolumn{4}{|l|}{ - $\geq$ Secondary } \\
\hline Marital status (n, \%) & $94(24.9)$ & $80(31.9)$ & 0.092 \\
\hline - Single & $144(38.1)$ & $78(31.1)$ & \\
\hline - Married & $140(37.0)$ & $93(37.1)$ & \\
\hline \multicolumn{4}{|c|}{ - Separated/Divorced/Widowed } \\
\hline Employment status (n, \%) & $53(14.0)$ & $60(23.9)$ & 0.002 \\
\hline - Unemployed & & & \\
\hline
\end{tabular}

*P-values presented are calculated using Mann Whitney $U$ or Kruskal Wallis tests as appropriate. Abbreviations: $n$, number; \%, percentage; SD, standard deviation; TSH, Tanzanian shilling; IQR, interquartile range; ART, antiretroviral treatment; VL, viral load 


\begin{tabular}{|c|c|c|c|}
\hline & $\begin{array}{l}\text { Clinic-based }(\mathrm{N}= \\
378)\end{array}$ & DSD $(N=251)$ & $\begin{array}{l}\text { p- } \\
\text { value* }\end{array}$ \\
\hline \multirow{5}{*}{$\begin{array}{l}\text { Income level (TSH), median (IQR) } \\
-<100,000 \\
-100,000-300,000 \\
->300,000\end{array}$} & $\begin{array}{l}87000(50000- \\
172000)\end{array}$ & $80000(50000-$ & \multirow[t]{5}{*}{0.315} \\
\hline & & & \\
\hline & $206(54.5)$ & 148 (59.0) & \\
\hline & $116(30.7)$ & $63(25.1)$ & \\
\hline & $56(14.8)$ & $40(15.9)$ & \\
\hline \multicolumn{4}{|l|}{ HIV care information } \\
\hline Years on ART, median [IQR] & $3.4[2.1-5.8]$ & $4.2[2.2-7.3]$ & 0.001 \\
\hline Years on ART group around mean & $219(59.4)$ & $130(53.3)$ & 0.161 \\
\hline$-\leq 4.4$ years & $150(40.6)$ & $114(46.7)$ & \\
\hline \multicolumn{4}{|l|}{$->4.4$ years } \\
\hline CD4 at ART start in cells $/ \mathrm{mm}^{3}$, median [IQR] & $273.5[155-449]$ & $340[184.5-513]$ & 0.003 \\
\hline CD4 at ART start groups & $126(34.8)$ & $63(26.7)$ & 0.045 \\
\hline$-<200$ & $236(65.2)$ & $173(73.3)$ & \\
\hline \multicolumn{4}{|l|}{$-\geq 200$} \\
\hline Viral load in copies/ml, median [IQR] & $10[10-10]$ & 10 [10-10] & 0.876 \\
\hline Viral load group & $375(99.2)$ & $237(94.4)$ & $<0.001$ \\
\hline$-<50$ copies $/ \mathrm{ml}$ & - & $9(3.6)$ & \\
\hline$-\geq 50$ & $3(0.8)$ & $5(2.0)$ & \\
\hline \multicolumn{4}{|l|}{ - missing } \\
\hline Time since last VL record, $\mathrm{n}(\%)$ & $179(47.4)$ & $113(45.0)$ & $<0.001$ \\
\hline$-\leq 6$ months & $170(44.9)$ & $85(33.9)$ & \\
\hline - 6 months -1 year & $26(6.9)$ & $49(19.5)$ & \\
\hline - > 1 year & $3(0.8)$ & $4(1.6)$ & \\
\hline \multicolumn{4}{|l|}{ - missing } \\
\hline \multicolumn{4}{|l|}{ Service access information } \\
\hline Time spent in clinic/club in minutes, median [IQR] & $115.2[57.6-172.8]$ & $43.2[28.8-115.2]$ & $<0.001$ \\
\hline Length of stay, n (\%) & $129(34.1)$ & $177(70.5)$ & $<0.001$ \\
\hline - Short ( $\leq 1 \mathrm{hr} 30 \mathrm{~min})$ & $246(65.1)$ & $71(28.3)$ & \\
\hline - Long (> 1hr 30 min) & & & \\
\hline
\end{tabular}

*P-values presented are calculated using Mann Whitney $\mathrm{U}$ or Kruskal Wallis tests as appropriate. Abbreviations: $\mathrm{n}$, number; \%, percentage; SD, standard deviation; TSH, Tanzanian shilling; IQR, interquartile range; ART, antiretroviral treatment; VL, viral load 


\begin{tabular}{|c|c|c|c|}
\hline & $\begin{array}{l}\text { Clinic-based (N= } \\
\text { 378) }\end{array}$ & $\mathrm{DSD}(\mathrm{N}=251)$ & $\begin{array}{l}\text { p- } \\
\text { value* }\end{array}$ \\
\hline \multirow{3}{*}{$\begin{array}{l}\text { Time spent travelling to clinic/club in minutes, } \\
\text { median [IQR] }\end{array}$} & $57.6[28.8-115.2]$ & $28.8[14.4-28.8]$ & $<0.001$ \\
\hline & $214(56.6)$ & $232(92.4)$ & $<0.001$ \\
\hline & $161(42.4)$ & $17(6.8)$ & \\
\hline \multicolumn{4}{|l|}{ Travel time group, $\mathrm{n}(\%)$} \\
\hline \multicolumn{4}{|l|}{ - Short ( $\leq 1 \mathrm{hr})$} \\
\hline \multicolumn{4}{|l|}{ - Long (> $\mathrm{hr}$ ) } \\
\hline Frequency of visits/meetings, $n$ (\%) & $355(93.9)$ & $121(48.2)$ & $<0.001$ \\
\hline - More ( $\leq$ every 2 months) & $23(6.1)$ & $130(51.8)$ & \\
\hline \multicolumn{4}{|l|}{ - Less (> every 2 months) } \\
\hline $\begin{array}{l}\text { *P-values presented are calculated using Mann } \\
\text { number; \%, percentage; SD, standard deviation; } \\
\text { treatment; } \mathrm{VL} \text {, viral load }\end{array}$ & $\begin{array}{l}\text { or Kruskal Wall } \\
\text { anian shilling: IC }\end{array}$ & $\begin{array}{l}\text { as appropriate. Abb } \\
\text { quartile range; } A R T \text {. }\end{array}$ & $\begin{array}{l}\text { ions: } \mathrm{n}_{\text {, }} \\
\text { troviral }\end{array}$ \\
\hline
\end{tabular}

\section{Fahi Total And Domain Scores By Service Delivery Model}

Clinic and DSD participants show comparable mean HRQoL scores across domains with only slight differences in the physical and emotional wellbeing domains (36.4 vs 35.5, max-40 $p<0.01)$ and $(32.1$ vs 32.8, max-40 $p<0.05)$

(Fig. 1a). No differences were observed in satisfactory HRQoL percentages across domains except for FGWB where more clinic participants revealed satisfactory HRQoL as compared to DSD. Satisfactory HRQoL overall was highest in the CF domain (89.2 vs 93.6) and lowest in the EWB (68.8 vs 68.5) and SWB (74.1 vs71.7) domains (Fig. 1b).

\section{Associations between sociodemographic, HIV-related and delivery mode-related factors and satisfactory overall HRQOL}

Satisfactory overall HRQoL was associated with being male ((prevalence ratio1.18, 95\% confidence interval 1.061.31), with being married (1.22, 1.02-1.47) and living in an urban setting $(1.28,1.09-1.49)$ in DSD care (see - Table 2). Less than satisfactory HRQoL was observed with increasing age among clinic participants, increasing income and increased meeting duration among DSD participants. HIV care factors were generally not associated with satisfactory overall HRQoL, while time spent during clinic/club was the only service access factor associated with satisfactory overall HRQoL.

Associations between sociodemographic, HIV care and service access factors and satisfactory domain HRQoL Compared to being single, satisfactory HRQoL was associated with being married in the PWB, FGWB and SWB domains among DSD participants. Being separated, divorced, or widowed was positively associated with satisfactory HRQoL for both clinic and DSD participants only in the SWB

domain. Living in an urban area was significantly associated with satisfactory HRQoL for both clinic and 
DSD participants in the EWB domains and only among DSD participants in the SWB and PWB domains. Less than satisfactory HRQoL was generally associated with declining age across domains but significant among clinic participants in the PWB, FGWB and SWB domains. In the EWB domain however, the reverse was observed among DSD participants across all age groups but significant with among those aged over 65 years. Surprisingly, less satisfactory HRQoL was also linked with increased income level in the PWB and EWB domain. Generally, HIV-related factors were not associated with satisfactory HRQoL. Among service delivery-related factors, DSD participants reported less than satisfactory HRQoL only for longer time spent during clinic/club in the PWB, EWB and SWB domains (Table 2 and Additional file 1 [for additional results of FGWB \& CF domains]). 
Table 2

Modified Poisson Regression with Robust Variance: Multivariable Association between Sociodemographic, HIV and Service delivery-related variables and satisfactory FAHI QoL scores\#

\begin{tabular}{|c|c|c|c|c|c|c|c|c|}
\hline & \multicolumn{2}{|c|}{ FAHltotal } & \multicolumn{2}{|l|}{ PWB } & \multicolumn{2}{|l|}{ EWB } & \multicolumn{2}{|l|}{ SWB } \\
\hline & Clinic & DSD & Clinic & DSD & Clinic & DSD & Clinic & DSD \\
\hline \multicolumn{9}{|l|}{ Covariates } \\
\hline \multicolumn{9}{|c|}{ Sociodemographic } \\
\hline Sex & Ref & Ref & Ref & Ref & Ref & Ref & Ref & Ref \\
\hline - Female & 1.18 & 1.03 & 1.00 & 0.94 & 1.09 & 0.93 & 1.18 & 1.14 \\
\hline - Male & $\begin{array}{l}\text { [1.06- } \\
1.31] \\
\star \star\end{array}$ & $\begin{array}{l}{[0.87-} \\
1.22]\end{array}$ & $\begin{array}{l}{[0.91-} \\
1.11]\end{array}$ & $\begin{array}{l}{[0.80-} \\
1.10]\end{array}$ & $\begin{array}{l}{[0.93-} \\
1.26]\end{array}$ & $\begin{array}{l}{[0.78-} \\
1.12]\end{array}$ & $\begin{array}{l}{[1.03-} \\
1.34]^{*}\end{array}$ & $\begin{array}{l}{[0.98-} \\
1.33]\end{array}$ \\
\hline \multirow{4}{*}{$\begin{array}{l}\text { Age } \\
-18-25 \\
-25-35 \\
-35-45\end{array}$} & Ref & Ref & Ref & Ref & Ref & Ref & Ref & Ref \\
\hline & 0.86 & 1.10 & 0.86 & 1.22 & 0.89 & 1.06 & 0.83 & 1.12 \\
\hline & $\begin{array}{l}{[0.74-} \\
0.99]^{\star}\end{array}$ & $\begin{array}{l}{[0.85-} \\
1.43]\end{array}$ & $\begin{array}{l}{[0.75-} \\
0.98]^{*}\end{array}$ & $\begin{array}{l}{[0.92-} \\
1.62]\end{array}$ & $\begin{array}{l}{[0.66-} \\
1.19]\end{array}$ & $\begin{array}{l}{[0.77-} \\
1.46]\end{array}$ & $\begin{array}{l}{[0.68-} \\
1.02]\end{array}$ & $\begin{array}{l}{[0.74-} \\
1.70]\end{array}$ \\
\hline & 0.77 & 1.11 & 0.86 & 1.23 & 0.89 & 1.23 & 0.73 & 1.07 \\
\hline $\begin{array}{l}-45-55 \\
-55-65\end{array}$ & $\begin{array}{l}{[0.66-} \\
0.90]^{* *}\end{array}$ & $\begin{array}{l}{[0.88-} \\
1.39]\end{array}$ & $\begin{array}{l}{[0.76-} \\
0.98]^{*}\end{array}$ & $\begin{array}{l}{[0.94-} \\
1.61]\end{array}$ & $\begin{array}{l}{[0.67-} \\
1.19]\end{array}$ & $\begin{array}{l}{[0.91-} \\
1.66]\end{array}$ & $\begin{array}{l}{[0.60-} \\
0.90]^{\star \star}\end{array}$ & $\begin{array}{l}{[0.72-} \\
1.59]\end{array}$ \\
\hline \multirow[t]{6}{*}{$->65$} & 0.73 & 0.95 & 0.86 & 1.06 & 0.82 & 1.98 & 0.77 & 0.94 \\
\hline & $\begin{array}{l}{[0.62-} \\
0.87] * *\end{array}$ & $\begin{array}{l}{[0.73-} \\
1.23]\end{array}$ & $\begin{array}{l}{[0.74-} \\
0.99] *\end{array}$ & $\begin{array}{l}{[0.78-} \\
1.44]\end{array}$ & $\begin{array}{l}{[0.60-} \\
1.12]\end{array}$ & $\begin{array}{l}{[0.71-} \\
1.33]\end{array}$ & $\begin{array}{l}{[0.63-} \\
0.96] *\end{array}$ & $\begin{array}{l}{[0.62-} \\
1.41]\end{array}$ \\
\hline & 0.77 & 0.97 & 0.91 & 1.17 & 0.96 & 1.02 & 0.81 & 1.08 \\
\hline & $\begin{array}{l}{[0.62-} \\
0.94]^{\star}\end{array}$ & $\begin{array}{l}{[0.72-} \\
1.31]\end{array}$ & $\begin{array}{l}{[0.77-} \\
1.07]\end{array}$ & $\begin{array}{l}{[0.87-} \\
1.59]\end{array}$ & $\begin{array}{l}{[0.68-} \\
1.34]\end{array}$ & $\begin{array}{l}{[0.70-} \\
1.49]\end{array}$ & $\begin{array}{l}{[0.61-} \\
1.07]\end{array}$ & $\begin{array}{l}{[0.70-} \\
1.67]\end{array}$ \\
\hline & 0.62 & 0.81 & 0.63 & 0.88 & 0.87 & 1.49 & 0.82 & 1.04 \\
\hline & $\begin{array}{l}{[0.39-} \\
1.00]^{*}\end{array}$ & $\begin{array}{l}{[0.51-} \\
1.28]\end{array}$ & $\begin{array}{l}{[0.41-} \\
0.99]^{*}\end{array}$ & $\begin{array}{l}{[0.54-} \\
1.43]\end{array}$ & $\begin{array}{l}{[0.54-} \\
1.39]\end{array}$ & $\begin{array}{l}{[1.02-} \\
2.18]^{\star}\end{array}$ & $\begin{array}{l}{[0.54-} \\
1.23]\end{array}$ & $\begin{array}{l}{[0.62-} \\
1.76]\end{array}$ \\
\hline \multirow{6}{*}{$\begin{array}{l}\text { Education } \\
\text { - None } \\
\text { - Primary } \\
\text { - } \geq \text { Secondary }\end{array}$} & Ref & Ref & Ref & Ref & Ref & Ref & Ref & Ref \\
\hline & 1.03 & 0.95 & 0.96 & 0.92 & 1.01 & 0.97 & 1.08 & 0.75 \\
\hline & $\begin{array}{l}{[0.92-} \\
1.17]\end{array}$ & $\begin{array}{l}{[0.79-} \\
1.14]\end{array}$ & $\begin{array}{l}{[0.87-} \\
1.06]\end{array}$ & $\begin{array}{l}{[0.78-} \\
1.08]\end{array}$ & $\begin{array}{l}{[0.86-} \\
1.18]\end{array}$ & $\begin{array}{l}{[0.81-} \\
1.16]\end{array}$ & $\begin{array}{l}{[0.93-} \\
1.25]\end{array}$ & $\begin{array}{l}{[0.63-} \\
0.89]^{\star \star}\end{array}$ \\
\hline & & & 0.87 & 1.11 & 0.96 & 1.28 & 1.03 & 0.87 \\
\hline & 0.91 & 1.18 & $\begin{array}{l}{[0.69-} \\
1.08]\end{array}$ & $\begin{array}{l}{[0.93-} \\
1.34]\end{array}$ & $\begin{array}{l}{[0.68-} \\
1.35]\end{array}$ & $\begin{array}{l}{[0.95-} \\
1.73]\end{array}$ & $\begin{array}{l}{[0.76-} \\
1.40]\end{array}$ & $\begin{array}{l}{[0.62-} \\
1.20]\end{array}$ \\
\hline & $\begin{array}{l}{[0.67-} \\
1.22]\end{array}$ & $\begin{array}{l}{[0.93-} \\
1.50]\end{array}$ & & & & & & \\
\hline
\end{tabular}




\begin{tabular}{|c|c|c|c|c|c|c|c|c|}
\hline \multirow{2}{*}{ Marital status } & \multicolumn{2}{|c|}{ FAHltotal } & \multicolumn{2}{|l|}{ PWB } & \multicolumn{2}{|l|}{ EWB } & \multicolumn{2}{|l|}{ SWB } \\
\hline & Ref & Ref & Ref & Ref & Ref & Ref & Ref & Ref \\
\hline -Single & 1.15 & 1.22 & 1.21 & 1.23 & 1.17 & 1.13 & 1.45 & 1.56 \\
\hline - Married & $\begin{array}{l}{[0.99-} \\
1.34]\end{array}$ & $\begin{array}{l}{[1.02-} \\
1.46]^{\star}\end{array}$ & \multirow{3}{*}{\multicolumn{2}{|c|}{$\begin{array}{ll}{[0.99 .1 .27]} & {[1.03-} \\
1.07 & 1.46]^{\star} \\
& 1.09 \\
{[0.95-} & \\
1.21] & {[0.92-} \\
& 1.29]\end{array}$}} & $\begin{array}{l}{[0.96-} \\
1.42]\end{array}$ & $\begin{array}{l}{[0.93-} \\
1.37]\end{array}$ & $\begin{array}{l}{[1.18-} \\
1.78]^{\star \star}\end{array}$ & $\begin{array}{l}{[1.23-} \\
1.98]^{\star \star}\end{array}$ \\
\hline \multirow[t]{2}{*}{ Separated/Divorced/Widow } & 1.13 & 1.13 & \multirow{2}{*}{$\begin{array}{l}{[0.95-} \\
1.21]\end{array}$} & & 1.05 & 1.00 & 1.25 & 1.60 \\
\hline & $\begin{array}{l}\text { [0.97- } \\
1.31]\end{array}$ & $\begin{array}{l}{[0.95-} \\
1.35]\end{array}$ & & & $\begin{array}{l}{[0.87-} \\
2.28]\end{array}$ & $\begin{array}{l}{[0.83-} \\
1.21]\end{array}$ & $\begin{array}{l}{[1.01-} \\
1.55]^{\star}\end{array}$ & $\begin{array}{l}{[1.27-} \\
2.02]^{\star *}\end{array}$ \\
\hline \multicolumn{3}{|l|}{ Employment } & Ref & \multicolumn{5}{|l|}{ Ref } \\
\hline \multicolumn{3}{|l|}{ - Unemployed } & 1.03 & \multicolumn{5}{|l|}{0.88} \\
\hline \multicolumn{3}{|l|}{ - Employed } & $\begin{array}{l}{[0.89-} \\
1.19]\end{array}$ & \multicolumn{5}{|l|}{$\begin{array}{l}{[0.77-} \\
0.99]^{\star}\end{array}$} \\
\hline \multirow{5}{*}{$\begin{array}{l}\text { Income level } \\
-<100,000 \\
-100,000-300,000 \\
->300,000\end{array}$} & Ref & Ref & & & Ref & \multicolumn{3}{|l|}{ Ref } \\
\hline & 0.87 & \multicolumn{3}{|l|}{0.82} & 0.81 & \multicolumn{3}{|l|}{0.88} \\
\hline & $\begin{array}{l}{[0.76-} \\
0.99]^{*}\end{array}$ & \multicolumn{3}{|l|}{$\begin{array}{l}{[0.68-} \\
0.99]^{\star}\end{array}$} & $\begin{array}{l}{[0.68-} \\
0.96]^{\star}\end{array}$ & \multicolumn{3}{|l|}{$\begin{array}{l}{[0.73-} \\
1.07]\end{array}$} \\
\hline & 1.01 & \multicolumn{3}{|l|}{0.77} & 0.82 & \multicolumn{3}{|l|}{0.79} \\
\hline & $\begin{array}{l}{[0.89-} \\
1.13]\end{array}$ & \multicolumn{3}{|l|}{$\begin{array}{l}{[0.61-} \\
0.98]^{\star}\end{array}$} & $\begin{array}{l}{[0.66-} \\
1.02]\end{array}$ & \multicolumn{3}{|l|}{$\begin{array}{l}{[0.62-} \\
1.00]\end{array}$} \\
\hline Location & Ref & Ref & Ref & Ref & Ref & Ref & Ref & Ref \\
\hline - Bugisi (Rural) & 1.12 & 1.28 & 1.13 & 1.18 & 1.40 & 1.39 & 1.00 & 1.25 \\
\hline - Ngokolo (Urban) & $\begin{array}{l}{[0.97-} \\
1.28]\end{array}$ & $\begin{array}{l}{[1.09-} \\
1.49]^{\star \star}\end{array}$ & $\begin{array}{l}{[1.02-} \\
1.25]^{\star}\end{array}$ & $\begin{array}{l}{[1.06-} \\
1.31]^{\star \star}\end{array}$ & $\begin{array}{l}{[1.22-} \\
1.61]^{\star \star}\end{array}$ & $\begin{array}{l}{[1.17-} \\
1.65]^{\star *}\end{array}$ & $\begin{array}{l}{[0.83-} \\
1.21]\end{array}$ & $\begin{array}{l}{[1.09-} \\
1.45]^{\star * *}\end{array}$ \\
\hline \multicolumn{9}{|c|}{ HIV care variables not associated with satisfactory HRQoL in bivariate analysis } \\
\hline \multicolumn{9}{|l|}{ Service access } \\
\hline Wait time mins & Ref & 0.66 & 0.98 & 0.82 & 0.90 & 0.35 & 0.93 & 0.65 \\
\hline$-<90 \min$ & 0.99 & \multirow{2}{*}{$\begin{array}{l}{[0.52-} \\
0.84]^{\star \star}\end{array}$} & \multirow{2}{*}{$\begin{array}{l}{[0.89-} \\
1.08]\end{array}$} & \multirow{2}{*}{$\begin{array}{l}{[0.69-} \\
0.97]^{*}\end{array}$} & {$[0.78-$} & {$[0.24-$} & {$[0.82-$} & {$[0.51-$} \\
\hline ->90 min & $\begin{array}{l}{[0.89-} \\
1.10]\end{array}$ & & & & & & & \\
\hline
\end{tabular}

\section{Legend}

${ }^{*} p<0.01 ;{ }^{*} p<<0.001$. Significant ratios in bold. \# See Additional file 1 for table with FGWB and CF domain results

Contribution of sociodemographic, HIV care and Service access factors to variance observed in HRQoL

Table 3 shows the contribution of sociodemographic, HIV care and service access factors to the variance observed in HRQoL scores. The analyses revealed that among clinic participants, the variance in overall HRQoL score FAHI total explainable by sociodemographic variables in the first step was $8.4 \%$. The addition of HIV care variables in the second step increased the variance explained to $12.4 \%$. Finally, service access variables in the third step brought the total to 
14.9\%. For DSD participants, the variance explained was $11.9 \%, 16.7 \%$ and $43.6 \%$ in the first, second and third steps, respectively. Across all domains, the variance in HRQoL explainable by the 3-step hierarchical model for clinic participants were modest (see Table 3). The highest was reported in the EWB domain i.e. $8.5 \%, 11.4 \%$ and $18.5 \%$ and lowest in the CF domain i.e. 5.9\%, 9.1\% and 9.8\% respectively. Much higher proportion of variance was explained in overall FAHI 43.5, PWB 30.2, EWB 53.4 and SWB 35.1 among DSD participants. Additional file 2 shows the details of the hierarchical linear regression with coefficients of all covariate in each step.

Table 3

Contribution of sociodemographic, HIV-related and Service delivery-related factors to variance observed in HRQoL scores

\begin{tabular}{|c|c|c|c|c|c|c|c|c|c|c|c|c|}
\hline \multicolumn{13}{|c|}{ Variance explained by three factor categories ( $N=$ clinic vs DSD) } \\
\hline & \multicolumn{2}{|c|}{ FAHltotal } & \multicolumn{2}{|l|}{ PWB } & \multicolumn{2}{|l|}{ EWB } & \multicolumn{2}{|l|}{ FGWB } & \multicolumn{2}{|l|}{ SWB } & \multicolumn{2}{|l|}{ CF } \\
\hline & Clinic & DSD & Clinic & DSD & Clinic & DSD & Clinic & DSD & Clinic & DSD & Clinic & DSD \\
\hline $\begin{array}{l}\text { *Step } \\
1 \mathrm{R}^{2}\end{array}$ & 0.102 & 0.229 & 0.103 & 0.205 & 0.085 & 0.184 & 0.119 & 0.222 & 0.118 & 0.217 & 0.059 & 0.058 \\
\hline $\begin{array}{l}(\mathrm{N}= \\
378 \\
\text { Vs } \\
251)\end{array}$ & & & & & & & & & & & & \\
\hline $\begin{array}{l}\text { ^Step } \\
2 \mathrm{R}^{2} \\
(\mathrm{~N}= \\
351 \\
\text { vs } \\
226)\end{array}$ & 0.145 & 0.289 & 0.146 & 0.243 & 0.114 & 0.268 & 0.169 & 0.253 & 0.139 & 0.258 & 0.091 & 0.086 \\
\hline $\begin{array}{l}\text { \#Step } \\
3 \mathrm{R}^{2} \\
\mathrm{~N}= \\
345 \\
\text { vs } \\
223\end{array}$ & 0.149 & 0.435 & 0.149 & 0.302 & 0.185 & 0.534 & 0.167 & 0.297 & 0.148 & 0.351 & 0.098 & 0.099 \\
\hline $\begin{array}{l}\text { AlC } \\
\text { Step } \\
3\end{array}$ & 8.803 & 8.692 & 5.969 & 6.077 & 6.603 & 6.415 & 6.258 & 6.63 & 6.723 & 6.523 & 4.034 & 4.081 \\
\hline
\end{tabular}

Legend: *Step 1 - Contribution of sociodemographic factors to variance observed; ^Step 2 - Contribution of HIV care factors to variance observed and ${ }^{\#}$ Step 3 - Contribution of service access variables to variance observed form Hierarchical Multiple Linear Regression. Additional file 2 shows the regression coefficients for variables included in the models in steps 1-3.

\section{Goodness Of Fit Statistics}

Tolerance values ranged from 0.16 to 0.84 while the VIF values were from 1.19 to 6.3 suggesting that multicollinearity had no impact on the variables included. The AUC for our Poisson regression model was 0.76 showing acceptable ability of our model to discriminate - the effective range is usually from 0.5 to 1 . There was no significant difference in mean FAHI total scores when the step 3 models in the hierarchical regression was compared with step 1 models. 


\section{Discussion}

Our study compared factors influencing HRQoL among stable ART clients accessing care at either HIV clinics or DSD clubs in the Shinyanga region of Tanzania. Majority of participants in our study rate their HRQoL relatively satisfactory. Our results revealed that service access factors contributed considerably to HRQoL among DSD participants. We found that time spent during clinic/club and the settings of service delivery were factors significantly associated with perceived HRQoL.

Understanding HRQoL in African studies is relevant in the current "Treat-all" and DSD era. Previous HRQoL studies mostly compared HIV positive and negative people and/or PLHIV not on and on ART (2)(9). Similar HRQoL among stable clients seen in our study strengthens the case for DSD which may likely impact positively on care delivery to unstable clients concurrently who are more likely to have special needs $(39,40)$. The complex effect of service access factors on overall HRQoL suggest that other non-measured factors are likely also to influence HRQoL.

Time spent during clinic/club was the main service access factor associated with HRQoL in our study followed by reduced frequency of meetings among DSD participants. We found that shorter time spent accessing service and less frequent visits were positively associated with HRQoL. This may reflect the value placed on time saved. Service access factors are more commonly studied in relation to patient satisfaction and retention in care than in HRQoL but both are likely to be related. In Malawi, another DSD model- six-monthly appointments with three monthly refills at the facility, showed reduced time spent in clinic as a favorable outcome predicting retention (41). Similarly, clients reported time saved for other activities as the greatest benefit derived from another DSD intervention in Uganda (42). Relatedly, reduced travel time has been identified as beneficial for DSD participants and enabling its success though it was not independently associated with HRQoL in our study $(43,44)$.

As per HRQoL domains, literature reveals that social and psychological/emotional domains score the lowest in most HRQoL studies among PLHIV (16,21,25,45-49) which is in line with our findings. Reasons adduced for this include stigma and discrimination due to fear and lack of awareness as HIV continues to isolate those infected from meaningful relationships. In our population of stable ART clients, variance explainable to service access factors was notably largest i.e. $53.4 \%$ in the EWB domain highlighting the significance of the contribution of DSD among participants who likely face different psychological, emotional and social dilemmas. $(16,17,46)$.

While our finding that being male was associated with a more satisfactory HRQoL aligns with evidence from Tanzania, Burkina Faso, Ghana and Ethiopia (18,50-52), other studies reveal no association or favor women $(22,23,53-55)$. Though these studies did not target stable clients in particular, they illustrate the complexity of associations between gender and HRQoL. These findings also inform implementers on areas to explore when developing interventions.

Similar to findings with gender, age reveals intricacies of associations in literature, showing evidence of declining HRQoL with age $(49,50,52,54)$ among PLHIV, as well as improvement or no association $(18,23,53)$. Our results showed a trend of declining HRQoL with age mainly among clinic participants. Among DSD participants, we found a positive association of age with the EWB domain suggesting a protective effect of DSD on EWB with increasing age. Older adults may enjoy fewer social ties than younger adults and thus reap a larger emotional benefit from DSD. As the PLHIV population on ART ages and comorbidities increase, the emotional support provided by DSD will become increasingly important and could serve as a springboard for additional outreach interventions.

Context such as place of residence has been shown to be associated with HRQoL in LMIC $(20,21)$. Our study showed that urban participants at Ngokolo had higher HRQoL scores across most domains than did their rural counterparts at

Page $13 / 21$ 
Bugisi. Better living conditions, greater awareness about HIV, and the anonymity people generally enjoy living in an urban setting likely creates a less-stigmatizing space for PLHIV. Our findings that educational level, employment and income level was not associated with HRQoL however differs from reports in the literature which associates a better HRQoL among PLHIV with a higher level of education $(18,20,48-50)$; with employment $(19,54,56)$ and relatedly to higher income levels $(19,57)$. The prevailing socio-economic circumstances which is similar among participants irrespective of setting may likely be the explanation and can inform the additional interventions to explore for higher impact.

Despite viral suppression, HIV infection ultimately predicts sup-optimal HRQoL $(9,25)$. The assumption of 'normalcy' in all areas as PLHIV attain viral suppression may be ambitious especially in the context of stigma, living in socioeconomically difficult circumstances or with other chronic illnesses. The need to do more in these areas has been advocated especially for PLHIV in the rural areas, for women and for adolescents and young people living with HIV (AYPLHIV) $(18,24,25,56,58)$.

\section{Strengths and limitations}

Our study is among few HRQoL studies conducted recently in SSA in the era of DSD. It provides useful insights into factors influencing HRQoL in an African population. Our participants were drawn from different geographical settings which mimics the reality of our population and generated valuable information about the impact of DSD in these settings. Though observational with known biases, the analytical design of our study allowed for comparisons that produced a rich resource useful for informing implementation and policy nonetheless.

Clinic participants were selected for stability as defined by the Tanzanian guideline at the time of data collection while DSD participants were assumed to be stable. This might have biased our results in favor of clinic participants, however viral load-related variables were similar in both groups and not independently associated with HRQoL in our study.

The project sites were mission clinics which may limit the generalizability of our findings. However, we might expect that larger differences in HRQoL scores would be found when comparing DSD and clinics outside mission hospital setting, as better funding and service which characterize our setting likely obscured the effect of DSD

\section{Conclusion}

Our results reveal a considerable contribution of service access factors to HRQoL especially in emotional wellbeing among DSD participants. This result is encouraging in that DSD does not appear to compromise HRQoL and could contribute to an improvement in the emotional wellbeing of patients. This highlights DSD as an acceptable option to explore for HIV care (59-62). While DSD shows promise in improving acceptability among patients and, therefore, sustainability of such services, our research highlights future research avenues to identify supporting interventions to improve other HRQoL domains among PLHIV. Service providers will need to engage PLHIV and the community at large to identify supporting interventions relevant for adapting acceptable DSD interventions to maximize its benefit. This research supports the call for monitoring HRQoL by extending the 909090 target with a fourth HRQoL-related 90 (11).

\section{Abbreviations}

ART - Anti-Retroviral Therapy

ARV - Antiretroviral

Page $14 / 21$ 
AUC - Area Under Curve

AYPLHIV - Adolescent and Young People Living with HIV

CF - Cognitive Functioning

$\mathrm{Cl}$ - Confidence Interval

CHW - Community Health Worker

CTC - Care and Treatment Centre

DSD - Differentiated Service Delivery

EWB - Emotional Wellbeing

FAHI - Functional Assessment of HIV Infection

FGWB - Functional and Global Well being

HIC - High Income Country

HIV - Human Immunodeficiency Syndrome

HCW - Health Care Worker

HRQoL - Health Related Quality of Life

LMIC - Low- and Middle-Income Country

NACP - National AIDS Control Program

NIMR - National Institute for Medical Research

MOHCDGEC - Ministry of Health, Community Development, Gender, Elderly and Children Tanzania

PLHIV - People Living with HIV

PWB - Physical Wellbeing

QoL - Quality of Life

ROC - Receiver Operative Characteristics

SD - Standard Deviation

SSA - Sub-Saharan Africa

SWB - Social Wellbeing

T\&T - Universal Test \& Treat

VL - Viral Load 
VLS - Viral Load Suppression

WHO - World Health Organization

\section{Declarations}

\section{Ethics Approval and Consent to Participate}

Ethics approval for this research study was obtained from NIMR i.e. NIMR/HQ/R.8c/Vol. I/674. Written consent was obtained from individuals who agreed to participate in the study using appropriate forms which had been approved for same as part of the ethics application.

\section{Consent for publication}

Not Applicable

\section{Availability of data and materials}

The dataset used and analyzed during the current study are available from the corresponding author on reasonable request

\section{Competing interests}

The authors declare that they have no competing interests.

\section{Funding}

The Shinyanga T\&T project is supported by Gilead Sciences Inc. ONE was supported by the Erasmus Mundus Joint Doctorate Trans Global Health Programme (EMJD-TGH) and the Amsterdam Institute for Global health and Development (AIGHD). The funders had no role in the design, plan for data collection, analysis, interpretation and writing of this article.

\section{Authors contribution}

ONE, GBG, SH, DN and TRW contributed to the conceptualization and design of the study. ONE conducted the field study and data collection. ONE was responsible for data analysis and interpretation with guidance from DN. ONE, GBG, SH, JdK, TRW were all involved in the interpretation of the results. TRW was responsible for the overall scientific management of the study. ONE wrote the initial draft of the manuscript. All authors contributed to drafts of the manuscript, read, and approved the final version.

\section{Acknowledgements}

The Shinyanga and Simiyu Test \& Treat program in Tanzania is supported by Gilead Sciences (USA) and the Diocese of Shinyanga through the Good Samaritan Foundation (Vatican). The implementation of the project is by Doctors with Africa CUAMM and the Diocese of Shinyanga within the framework set out in the national guidance of the Tanzanian Ministry of Health, Community Development, Gender, Elderly and Children (MoHCDGEC) through the National AIDS Control Program (NACP). The scientific evaluation of the project is under the guidance of Principal Investigators Prof Anton Pozniak and Dr Bernard Desderius and is performed by the Amsterdam Institute for Global Health and Development (AIGHD) in collaboration with Doctors with Africa CUAMM. The content of this manuscript is solely the 
responsibility of the authors and does not necessarily represent the official views of any of the institutions mentioned above. We thank all our institutional collaborators, the study participants, the staff at the project clinical sites and laboratories, as well as the project support staff for their invaluable support to this program in general and the current manuscript in particular.

\section{References}

1. Louwagie GM, Bachmann MO, Meyer K, Booysen F le R, Fairall LR, Heunis C. Highly active antiretroviral treatment and health related quality of life in South African adults with human immunodeficiency virus infection: A crosssectional analytical study. BMC Public Health. 2007 Sep 14;7:244.

2. Thomas R, Burger R, Harper A, Kanema S, Mwenge L, Vanqa N, et al. Differences in health-related quality of life between HIV-positive and HIV-negative people in Zambia and South Africa: a cross-sectional baseline survey of the HPTN 071 (PopART) trial. Lancet Glob Heal. 2017;5(11):e1133-41.

3. Nakagawa F, May M, Phillips A. Life expectancy living with HIV. Curr Opin Infect Dis. 2013 Feb;26(1):17-25.

4. Pozniak A. Quality of life in chronic HIV infection. 2014;

5. Yang HY, Beymer MR, Suen S chuan. Chronic Disease Onset Among People Living with HIV and AIDS in a Large Private Insurance Claims Dataset. Sci Rep. 2019 Dec 1;9(1).

6. Schouten J, Wit FW, Stolte IG, Kootstra NA, Van Der Valk M, Geerlings SE, et al. Cross-sectional Comparison of the Prevalence of Age-Associated Comorbidities and Their Risk Factors Between HIV-Infected and Uninfected Individuals: The AGE h IV Cohort Study. 2014;

7. Friedman EE, Duffus WA. Chronic health conditions in Medicare beneficiaries 65 years and older with HIV infection HHS Public Access. AIDS. 2016;30(16):2529-36.

8. Mayer KH, Loo S, Crawford PM, Crane HM, Leo M, Denouden P, et al. Excess clinical comorbidity among HIVinfected patients accessing primary care in US community health centers. Public Health Rep. 2018 Jan 1;133(1):109-18.

9. Miners A, Phillips A, Kreif N, Rodger A, Speakman A, Fisher M, et al. Health-related quality-of-life of people with HIV in the era of combination antiretroviral treatment: A cross-sectional comparison with the general population. Lancet HIV. 2014;1(1):e32-40.

10. UNAIDS. 90-90-90 An ambitious treatment target to help end the AIDS epidemic. 2014.

11. Lazarus J V., Safreed-Harmon K, Barton SE, Costagliola D, Dedes N, del Amo Valero J, et al. Beyond viral suppression of HIV - the new quality of life frontier. Vol. 14, BMC Medicine. 2016. p. 94.

12. Karimi M, Brazier J. Health, Health-Related Quality of Life, and Quality of Life: What is the Difference? Pharmacoeconomics. 2016;34(7):645-9.

13. Romero M, Vivas-Consuelo D, Alvis-Guzman N. Is Health Related Quality of Life (HRQoL) a valid indicator for health systems evaluation? Springerplus. 2013 Dec 11;2(1):664.

14. Jelsma J, MacLean E, Hughes J, Tinise X, Darder M. An investigation into the health-related quality of life of individuals living with HIV who are receiving HAART. AIDS Care. 2005 Jul 18;17(5):579-88.

15. Robberstad B, Olsen J. The health related quality of life of people living with HIV/AIDS in sub-Saharan Africa - a literature review and focus group study. Cost Eff Resour Alloc. 2010;8(1):5.

16. Akinboro AO laday., Akinyemi SO molol., Olaitan PB, Raji AA deniy., Popoola AA detay., Awoyemi OR oselin., et al. Quality of life of Nigerians living with human immunodeficiency virus. Pan Afr Med J. 2014;18:234. 
17. Bello SI, Bello IK. Quality of life of HIV/AIDS patients in a secondary health care facility, llorin, Nigeria. Vol. 26, Proceedings (Baylor University. Medical Center). Baylor Health Care System; 2013. 116-9 p.

18. Bakiono F, Ouédraogo L, Sanou M, Samadoulougou S, Guiguemdé PWL, Kirakoya-Samadoulougou F, et al. Quality of life in people living with HIV: A cross-sectional study in Ouagadougou, Burkina Faso. Springerplus. 2014;3(1):1-11.

19. Oliveira FBM, Moura MEB, De Araújo TME, Andrade EMLR. Quality of life and associated factors in people living with HIV/AIDS. ACTA Paul Enferm. 2015;28(6):510-6.

20. Yaya I, Djalogue L, Patassi AA, Landoh DE, Assindo A, Nambiema A, et al. Health-related quality of life among people living with HIV/AIDS in Togo: Individuals and contextual effects. BMC Res Notes. 2019 Mar 15;12(1).

21. Imam M, Karim M, Ferdous C, Akhter S. Health related quality of life among the people living with HIV. Bangladesh Med Res Counc Bull. 1970 Jan 1;37(1):1-6.

22. Osei-Yeboah J, Owiredu WKBA, Norgbe GK, Lokpo SY, Obirikorang C, Allotey EA, et al. Quality of Life of People Living with HIV/AIDS in the Ho Municipality, Ghana: A Cross-Sectional Study. AIDS Res Treat. 2017;2017.

23. Erdbeer G, Sabranski M, Sonntag I, Stoehr A, Horst H-A, Plettenberg A, et al. Everything fine so far? Physical and mental health in HIV-infected patients with virological success and long-term exposure to antiretroviral therapy. $J$ Int AIDS Soc. 2014;17(November):19673.

24. Magafu MGMD, Moji K, Igumbor EU, Hashizume M, Mizota T, Komazawa O, et al. Usefulness of Highly Active Antiretroviral Therapy on Health-Related Quality of Life of Adult Recipients in Tanzania. AIDS Patient Care STDS. 2009 Jul;23(7):563-70.

25. Zeluf-Andersson G, Eriksson LE, Schönnesson LN, Höijer J, Månehall P, Ekström AM. Beyond viral suppression: the quality of life of people living with HIV in Sweden. AIDS Care - Psychol Socio-Medical Asp AIDS/HIV. 2019 Apr 3;31(4):403-12.

26. Donald KA. Health-related quality of life of people with HIV in Zambia and South Africa. 2017;

27. Parcesepe AM, Nash D, Tymejczyk O, Reidy W, Kulkarni SG, Elul B. Gender, HIV-Related Stigma, and Health-Related Quality of Life Among Adults Enrolling in HIV Care in Tanzania. AIDS Behav. 2019;

28. Bulali RE, Kibusi SM, Mpondo BCT. Factors Associated with HIV Status Disclosure and Its Effect on Treatment Adherence and Quality of Life among Children 6-17 Years on Antiretroviral Therapy in Southern Highlands Zone, Tanzania: Unmatched Case Control Study. Int J Pediatr. 2018 Jun 26;2018:1-10.

29. IAS. Differentiated Care for Hiv: a Decision Framework for Antiretroviral Therapy Delivery. Differ Care.org (International AIDS Soc. 2016;1-56.

30. UNAIDS. United Republic of Tanzania | UNAIDS [Internet]. Country Fact Sheet. 2018 [cited 2019 Nov 21]. Available from: http://www.unaids.org/en/regionscountries/countries/unitedrepublicoftanzania

31. Tsondai PR, Wilkinson LS, Grimsrud A, Mdlalo PT, Ullauri A, Boulle A. High rates of retention and viral suppression in the scale-up of antiretroviral therapy adherence clubs in Cape Town, South Africa. J Int AIDS Soc. 2017;20(Suppl 4):21649.

32. Okoboi S, Ding E, Persuad S, Wangisi J, Birungi J, Shurgold S, et al. Community-based ART distribution system can effectively facilitate long-term program retention and low-rates of death and virologic failure in rural Uganda. AIDS Res Ther. 2015;12(12).

33. Jaffar S, Amuron B, Foster S, Birungi J, Levin J, Namara G, et al. Rates of virological failure in patients treated in a home-based versus a facility-based HIV-care model in Jinja, southeast Uganda: a cluster-randomised equivalence trial. Lancet. 2009/11/27. 2009;374(9707):2080-9. 
34. Ministry of Health Community Development Gender Elderly and Children. National AIDS Control Programme Tanzania. NATIONAL GUIDELINES FOR THE MANAGEMENT OF HIV AND AIDS Sixth Edition October 2017. 2017; (October).

35. Nyongesa MK, Sigilai A, Hassan AS, Thoya J, Odhiambo R, Van de Vijver FJR, et al. A mixed methods approach to adapting and evaluating the functional assessment of HIV infection (FAHI), Swahili version, for use with low literacy populations. PLoS One. 2017;12(4):e0175021.

36. Cella D. Functional Assessment of HIV Infection. Facit. 2007;(November):1-4.

37. EpiData Software - http://www.epidata.dk [Internet]. [cited 2020 Feb 24]. Available from: https://www.epidata.dk/

38. Barros AJD, Hirakata VN. Alternatives for logistic regression in cross-sectional studies: an empirical comparison of models that directly estimate the prevalence ratio. BMC Med Res Methodol. 2003 Oct 20;3:21.

39. World Health Organization. Guidelines for managing advanced HIV disease and rapid initiation of antiretroviral therapy. Licence: CC BY-NC-SA 3.0 IGO. 2017;(July):1-56.

40. International AIDS Society. Differentiated care for HIV: a decision framework for antiretroviral therapy delivery for children, adolescents and pregnant and breastfeeding women. 2016.

41. McGuire M, Pedrono G, Mukhuna B, Huckabee M, Heinzelmann A, Szumilin E, et al. Category: D1 Strategies for increasing efficiency of service delivery, evaluation of different models of service delivery Title: Optimizing patient monitoring after the first year of ART: three years of implementing 6-monthly clinical appointments in rural Malawi.

42. Nakiwogga-Muwanga A, Katabira E, Sempa J, Kambugu A, Nakibuuka-Lubwama E, Lamorde M, et al. A Pharmacy-Only Refill Program at a Large HIV Clinic in Uganda: Experience and Satisfaction of Patients.

43. Bock P, Gunst C, Maschilla L, Holtman R, Grobbelaar N, Wademan D, et al. Retention in care and factors critical for effectively implementing antiretroviral adherence clubs in a rural district in South Africa. J Int AIDS Soc. 2019 Oct $6 ; 22(10)$.

44. MSF, SAMU. Reaching Closer to Home Progress implementing community-based and other adherence strategies supporting people on HIV treatment. Doctors Without Borders. 2013.

45. Fatiregun A, Mofolorunsho K, Osagbemi K. Quality Of Life Of People Living With Hiv/Aids In Kogi State, Nigeria. Benin J Postgrad Med. 2009 Dec 9;11(1).

46. Giri S, Neupane M, Pant S, Timalsina U, Koirala S, Timalsina S, et al. Quality of life among people living with acquired immune deficiency syndrome receiving anti-retroviral therapy: A study from Nepal. HIV/AIDS - Res Palliat Care. 2013 Sep 25;5:277-82.

47. Folasire OF, Irabor AE, Folasire AM. Quality of life of people living with HIV and AIDS attending the antiretroviral clinic, university college hospital, Nigeria. African J Prim Heal Care Fam Med. 2012;4(1).

48. Shan D, Ge Z, Ming S, Wang L, Sante M, He W, et al. Quality of life and related factors among HIV-positive spouses from serodiscordant couples under antiretroviral therapy in Henan Province, China. PLoS One. 2011;6(6).

49. Liping M, Peng X, Haijiang L, Lahong J, Fan L. Quality of life of people living with HIV/ AIDS: A Cross-sectional study in zhejiang province, China. PLoS One. 2015 Aug 26;10(8).

50. Abrefa-Gyan T, Cornelius LJ, Okundaye J. Socio-demographic factors, social support, quality of life, and HIV/AIDS in Ghana. J Evidence-Informed Soc Work. 2016;13(2):206-16.

51. Tesfay A, Gebremariam A, Gerbaba M, Abrha H. Gender differences in health related quality of life among people living with HIV on highly active antiretroviral therapy in mekelle town, northern ethiopia. Biomed Res Int. 2015 Jan $6 ; 2015$. 
52. Mwanyangala MA, Mayombana C, Urassa H, Charles J, Mahutanga C, Abdullah S, et al. Health status and quality of life among older adults in rural Tanzania. Glob Health Action. 2010 Sep 27;3.

53. Zeluf-Andersson G, Eriksson LE, Schönnesson LN, Höijer J, Månehall P, Ekström AM. AIDS Care Psychological and Socio-medical Aspects of AIDS/HIV Beyond viral suppression: the quality of life of people living with HIV in Sweden Beyond viral suppression: the quality of life of people living with HIV in Sweden. 2018;

54. M.H. I, M.R. K, C. F, S. A. Health related quality of life among the people living with HIV. Bangladesh Med Res Counc Bull. 2011;37(1):1-6.

55. Stangl AL, Bunnell R, Wamai N, Masaba H, Mermin J. Measuring quality of life in rural Uganda: Reliability and validity of summary scores from the Medical Outcomes Study HIV Health Survey (MOS-HIV). Qual Life Res. 2012 Nov;21(9):1655-63.

56. Mutabazi-Mwesigire D, Katamba A, Martin F, Seeley J, Wu AW. Factors that affect quality of life among people living with HIV attending an urban clinic in Uganda: A cohort study. PLoS One. 2015;10(6):1-21.

57. Mafirakureva N, Dzingirai B, Postma MJ, van Hulst M, Khoza S. Health-related quality of life in HIV/AIDS patients on antiretroviral therapy at a tertiary care facility in Zimbabwe. AIDS Care - Psychol Socio-Medical Asp AIDS/HIV. 2016;28(7):904-12.

58. Oguntibeju 00. Quality of life of people living with HIV and AIDS and antiretroviral therapy. Vol. 4, HIV/AIDS Research and Palliative Care. 2012. p. 117-24.

59. Schadé A, van Grootheest G, Smit JH. HIV-infected mental health patients: characteristics and comparison with HIV-infected patients from the general population and non-infected mental health patients. BMC Psychiatry. 2013 Dec 23;13(1):35.

60. Charles B, Jeyaseelan L, Pandian AK, Sam AE, Thenmozhi M, Jayaseelan V. Association between stigma, depression and quality of life of people living with HIV/AIDS (PLHA) in South India - a community based cross sectional study. BMC Public Health. 2012 Dec 21;12(1):463.

61. Simbayi LC, Kalichman S, Strebel A, Cloete A, Henda N, Mqeketo A. Internalized stigma, discrimination, and depression among men and women living with HIV/AIDS in Cape Town, South Africa. Soc Sci Med. 2007 May;64(9):1823-31.

62. Basha EA, Derseh BT, Haile YGE, Tafere G. Factors Affecting Psychological Distress among People Living with HIV/AIDS at Selected Hospitals of North Shewa Zone, Amhara Region, Ethiopia. AIDS Res Treat. 2019;2019.

\section{Figures}




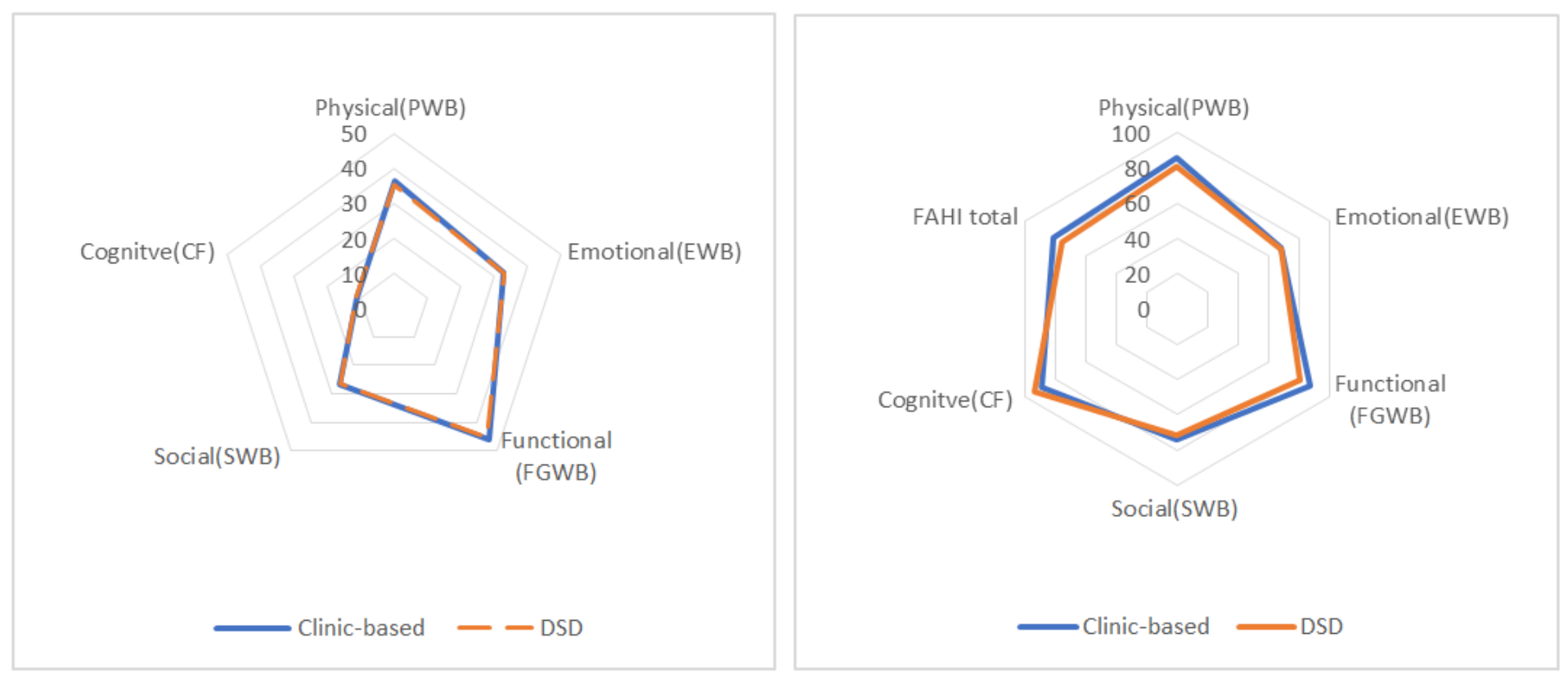

Figure 1

FAHI HRQoL scores by delivery model Abbreviations: PWB, Physical wellbeing; EWB, Emotional wellbeing; FGWB, Functional and Global wellbeing; SWB, Social wellbeing; CF, Cognitive Functioning

\section{Supplementary Files}

This is a list of supplementary files associated with this preprint. Click to download.

- 2020.09.14QualityoflifeTnTShinyangaAdditionalfile1.xIsx

- 2020.09.14QualityoflifeTnTShinyangaAdditionalfile2.xIsx 